\title{
Clinical outcomes and healthcare costs of inpatients with tetanus in Korea, 2011- 2019
}

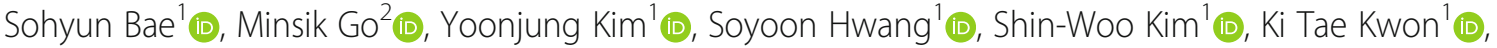 \\ Sook-In Jung ${ }^{3 * \dagger}$ (D) and Hyun-Ha Chang ${ }^{1 *+}$ (D)
}

\begin{abstract}
Background: Tetanus is a rare, vaccine-preventable but extremely serious disease. We investigated the recent trend of the clinical outcomes and medical costs for inpatients with tetanus in South Korea over 10 years.

Methods: We conducted a retrospective review to determine the clinical factors and medical costs associated with tetanus at two national university hospitals in South Korea between January 2011 and October 2019.

Results: Forty-nine patients were admitted for tetanus (mean age, 67.0 years [range, 53.0-80.0 years]; 32 women [57.1\%]). All the patients had generalized tetanus, and 5 (10.2\%) died during hospitalization. The median duration from symptom onset to hospital admission was 4 days. Trismus (85.7\%) was the most common symptom, and the median hospital stay was 39 days. Thirty-two patients (65.3\%) required mechanical ventilation, and 20 (40.8\%) developed aspiration pneumonia. The median total healthcare cost per patient was US $\$ 18,011$. After discharge, 35 patients (71.4\%) recovered sufficiently to walk without disability.

Conclusions: Tetanus requires long hospital stays and high medical expenditures in South Korea; however, the vaccination completion rate is low. Medical staff should therefore promote medical advice and policies on the management of tetanus to the general South Korean population.
\end{abstract}

Keywords: Tetanus, Healthcare costs, Treatment outcome

\section{Background}

Tetanus is a fatal but preventable infectious disease caused by Clostridium tetani. According to the US Centers for Disease Control and Prevention, tetanus vaccines have largely contributed to the reduced tetanus surveillance and morbidity since 1990 [1]. Similarly, the

\footnotetext{
* Correspondence: sijung@chonnam.ac.kr; changhha@knu.ac.kr

${ }^{\dagger}$ Hyun-Ha Chang and Sook-In Jung are authors contributed equally to this study.

${ }^{3}$ Department of Infectious Diseases, Chonnam National University Medical School, 42 Jebongro, Donggu, Gwangju 61469, South Korea

'Division of Infectious Diseases, Department of Internal Medicine, School of Medicine, Kyungpook National University, 130 Dongdeok-ro, Jung-gu, Daegu 41944, South Korea

Full list of author information is available at the end of the article
}

incidence of tetanus in South Korea has decreased since the introduction of the diphtheria and tetanus toxoid and acellular pertussis vaccine in 1982 [2].

As recommended by the Committee on Infectious Disease of the Korean Pediatric Society, the tetanus immunization schedule for children is five doses of diphtheria and tetanus toxoids and acellular pertussis vaccine at $2,4,6,15$, and 18 months of age, and then again at 46 years. This schedule should be followed by a tetanus toxoid, diphtheria, and acellular pertussis (Tdap) vaccine dose at 11-12 years of age, and a tetanus toxoid vaccine (Td) dose every 10 years [3]. However, insufficient tetanus vaccinations have been administered to adults in certain cases of tetanus. According to the Korea Centers

(c) The Author(s). 2021 Open Access This article is licensed under a Creative Commons Attribution 4.0 International License, which permits use, sharing, adaptation, distribution and reproduction in any medium or format, as long as you give appropriate credit to the original author(s) and the source, provide a link to the Creative Commons licence, and indicate if changes were made. The images or other third party material in this article are included in the article's Creative Commons licence, unless indicated otherwise in a credit line to the material. If material is not included in the article's Creative Commons licence and your intended use is not permitted by statutory regulation or exceeds the permitted use, you will need to obtain permission directly from the copyright holder. To view a copy of this licence, visit http://creativecommons.org/licenses/by/4.0/. The Creative Commons Public Domain Dedication waiver (http://creativecommons.org/publicdomain/zero/1.0/) applies to the data made available in this article, unless otherwise stated in a credit line to the data. 
for Disease Control and Prevention (KCDC), the nationwide incidence of tetanus increased to a mean of 23.9 cases per year between 2010 and 2019 as compared with the mean of 10.9 cases per year between 2000 and 2009 . Given the steady increase in its incidence, tetanus remains difficult to prevent and treat. Unfortunately, studies are limited regarding tetanus cases in South Korea. The most recent study on the clinical manifestations of tetanus was conducted at Chonnam National University in 2003. It focused on the clinical and epidemiological findings of tetanus from 17 cases in South Korea, with no mention regarding the cost incurred for the treatment of the disease. We therefore aimed to investigate the clinical features and inpatient costs of tetanus within the past 10 years.

\section{Methods}

\section{Data collection}

This retrospective cohort study was conducted between January 1, 2011 and July 30, 2019 at Kyungpook National University Hospital and Chonnam National University Hospital. The definition of patients with tetanus was extracted from the nomenclature of the International Classification of Disease, Tenth Revision (ICD10). Two physicians reviewed the patient data that included demographics, clinical characteristics, and laboratory findings retrieved from the hospital medical records and data. All the included patients were older than 18 years and diagnosed as having tetanus at 1 of 2 tertiary referral centers. We did not include neonatal tetanus. Tetanus was defined according to the clinical features, such as generalized or localized muscular spasm and contractures without other medical causes. As defined in this study, generalized tetanus is characterized by painful muscle spasms of the jaw and neck, muscular contraction unrelated to injury sites, or autonomic dysfunction, and patients who presented fluctuating systolic blood pressure or heart rate [4] or required inotropic agents or calcium channel blockers to control their blood pressure were defined as having autonomic dysfunction. The patients were divided into two groups, a non-mechanical ventilation (nonMV) group and a mechanical ventilation (MV) group. The MV group included patients who received invasive ventilation during hospitalization, while the nonMV group included patients who were not administered ventilation. We collected data on the patients' demographic characteristics, tetanus-related clinical features, hospital stay duration, rehabilitation treatment duration, infection source, complications during treatment, and medical costs for tetanus treatment. Aspiration pneumonia was confirmed by a radiologist by performing chest radiography or CT scans, the results of which were reviewed by a physician. The requirement for rehabilitation was determined by consultation with the rehabilitation professionals. We considered loss of immunization visits when the patients did not attend the arranged outpatient clinic visit on time and no medical referral was indicated in the electronic medical records. We also collected data on the laboratory results and expenditures during the tetanus treatment. The medical charts from most of the patients were reviewed during their first and last hospital visits.

\section{Statistical analyses}

All statistical data were analyzed using $\mathrm{R}$ statistics version 3.1. The categorical variables are expressed as numbers and percentages and were compared using the chisquare test or Fisher exact test. The continuous variables are expressed as mean \pm standard deviation (SD) or median with the interquartile range (IQR) and were compared using the Mann-Whitney $U$ test. Data that did not exhibit a normal distribution are represented as median with IQR. We used a multivariate logistic regression analysis to evaluate the risk factors associated with MV. $P$ values $<0.05$ were considered statistically significant.

\section{Ethics statement}

The institutional review board of Kyungpook National University Hospital and Chonnam National University Hospital reviewed and approved our study protocol (approval Nos. KNUH-201907031 and CNUH-2019235, respectively). Considering the retrospective nature of the study and the use of anonymous clinical data for the analysis, the requirement for informed consent was waived.

\section{Results}

\section{Patient characteristics and clinical features}

We identified 49 patients aged $\geq 18$ years who were hospitalized for tetanus, of whom 32 required invasive MV. Table 1 lists the baseline characteristics and clinical presentation of the MV and nonMV groups. Among the 49 patients with tetanus, 32 (57.1\%) were women, and the median age was 67.0 years (IQR, 53.0-80.0). The demand for MV increased significantly among the older patients (median age, 72.5 years [IQR, 59.0-82.0 years] and 53.0 years [IQR, 46.0-67.0 years] for the MV and nonMV groups, respectively; $P=0.002$ ). The incubation time was shorter for the nonMV group than for the MV group but did not differ significantly (median, 6.0 days [IQR, 3.0-8.0 days] vs. 2.0 [IQR, $1.0-6.0$ days]; $P=$ 0.237 ). All the patients developed generalized tetanus. With regard to the entry site of the toxin, an unknown entry site $(34.7 \%)$ was the most common, followed by the legs (24.5\%) and arms (22.4\%). The most common symptoms on admission were trismus (85.7\%) and muscle stiffness (73.5\%). Most patients developed 
Table 1 Characteristics of the patients with tetanus according to the mechanical ventilation required

\begin{tabular}{|c|c|c|c|c|}
\hline Factors & $\begin{array}{l}\text { Total }(n= \\
\text { 49) }\end{array}$ & $\begin{array}{l}\text { No mechanical ventilation } \\
(n=17)\end{array}$ & $\begin{array}{l}\text { Mechanical ventilation } \\
(n=32)\end{array}$ & $\begin{array}{l}P \\
\text { value }\end{array}$ \\
\hline \multicolumn{5}{|l|}{ General characteristics } \\
\hline Women, $n(\%)$ & $32(57.1 \%)$ & $11(64.7 \%)$ & $21(65.6 \%)$ & 0.949 \\
\hline Median age, years [IQR] & $\begin{array}{l}67.0[53.0 ; \\
80.0]\end{array}$ & $53.0[46.0 ; 67.0]$ & $72.5[59.0 ; 82.0]$ & 0.002 \\
\hline Median incubation time, days [IQR] & $5.0[1.5-7.5]$ & $2.0[1.0-6.0]$ & $6.0[3.0-8.0]$ & 0.237 \\
\hline $\begin{array}{l}\text { Median duration from symptom onset to admission, } \\
\text { days [IQR] }\end{array}$ & $4.0[2.0-9.0]$ & $4.0[2.0-6.5]$ & $7.0[5.0-1.5]$ & 0.120 \\
\hline Wound operation & $6(12.2 \%)$ & $3(18.8 \%)$ & $3(12.5 \%)$ & 0.928 \\
\hline \multicolumn{5}{|l|}{ Comorbidity, $n$ (\%) } \\
\hline Hypertension & $16(32.7 \%)$ & $3(17.6 \%)$ & $13(40.6 \%)$ & 0.189 \\
\hline Diabetes mellitus & $11(22.4 \%)$ & $3(17.6 \%)$ & $8(25.0 \%)$ & 0.820 \\
\hline Malignancy & $8(16.3 \%)$ & $5(29.4 \%)$ & $3(9.4 \%)$ & 0.161 \\
\hline Others & $17(34.7 \%)$ & $4(23.5 \%)$ & $13(40.6 \%)$ & 0.378 \\
\hline Site of entry, $n(\%)$ & & & & 0.314 \\
\hline Legs & $12(24.5 \%)$ & $5(29.4 \%)$ & $7(21.9 \%)$ & \\
\hline Arms & $11(22.4 \%)$ & $5(29.4 \%)$ & $6(18.8 \%)$ & \\
\hline Face & $7(14.3 \%)$ & $7(15.9 \%)$ & 0 & \\
\hline Trunk & $7(14.3 \%)$ & $0(0.0 \%)$ & $7(21.9 \%)$ & \\
\hline Cryptogenic & $17(34.7 \%)$ & $6(35.3 \%)$ & $11(34.4 \%)$ & \\
\hline \multicolumn{5}{|l|}{ Symptom during hospitalization, $\boldsymbol{n}(\%)$} \\
\hline Trismus & $42(85.7 \%)$ & $14(82.4 \%)$ & $28(87.5 \%)$ & 0.951 \\
\hline Muscle stiffness & $36(73.5 \%)$ & $7(41.2 \%)$ & $29(90.6 \%)$ & 0.001 \\
\hline Swallowing difficulty & $33(67.3 \%)$ & $9(52.9 \%)$ & $24(75.0 \%)$ & 0.212 \\
\hline Dysarthria & $32(65.3 \%)$ & $11(64.7 \%)$ & $21(65.6 \%)$ & 1.000 \\
\hline Opisthotonos & $11(22.4 \%)$ & $2(11.8 \%)$ & $9(28.1 \%)$ & 0.344 \\
\hline Seizure & $11(22.4 \%)$ & $2(11.8 \%)$ & $9(28.1 \%)$ & 0.344 \\
\hline Fever & $25(51.0 \%)$ & $5(29.4 \%)$ & $20(62.5 \%)$ & 0.057 \\
\hline Nausea and vomiting & $2(4.1 \%)$ & $1(5.9 \%)$ & $1(3.1 \%)$ & 1.000 \\
\hline Sialorrhea & $2(4.1 \%)$ & - & $2(6.2 \%)$ & 0.769 \\
\hline Dyspnea & $28(57.1 \%)$ & $5(29.4 \%)$ & $23(71.9 \%)$ & 0.011 \\
\hline Autonomic dysfunction & $43(87.8 \%)$ & $11(64.7 \%)$ & $32(100 \%)$ & 0.002 \\
\hline
\end{tabular}

Abbreviation: $N$ number, $I Q R$ interquartile range

autonomic nervous dysfunction, and half of the patients $(51.0 \%)$ developed fever during their hospital stay.

\section{Clinical findings of the patients who died}

The median age of the five patients who died was 85 years (range, $77-89$ years), and four (80\%) of these patients were women. Four of the five patients had no definitive injuries and did not know the incubation time. In one case, the dorsum of the right hand was injured 14 days prior to symptom onset. Before admission, none of the five patients remembered their tetanus vaccination history. Four patients were provided with MV, and one patient refused life-sustaining treatment, including MV and cardiopulmonary resuscitation because of their advanced age. The cause of death in all five patients was respiratory failure. The tetanus complications in this group included aspiration pneumonia in two patients, pulmonary thromboembolism in one patient, and multifocal infarction in one patient.

\section{Clinical outcomes and significant factors of mechanical ventilation status}

Table 2 provides the details of the clinical outcomes and complications. Twenty patients (40.8\%) experienced aspiration pneumonia, and $18(36.7 \%)$ had concurrent ventilator-associated pneumonia. Twenty-one (65.6\%) cases of nosocomial infection were reported in the MV 
Table 2 Clinical outcomes of the patients with tetanus according to the mechanical ventilation required

\begin{tabular}{|c|c|c|c|c|}
\hline Factors & $\begin{array}{l}\text { Total }(n= \\
49)\end{array}$ & $\begin{array}{l}\text { No mechanical ventilation }(n= \\
\text { 17) }\end{array}$ & $\begin{array}{l}\text { Mechanical ventilation }(n= \\
\text { 32) }\end{array}$ & $\begin{array}{l}P \\
\text { value }\end{array}$ \\
\hline \multicolumn{5}{|l|}{ Complications, $n$ (\%) } \\
\hline Aspiration pneumonia & $20(40.8 \%)$ & $2(11.8 \%)$ & $18(56.2 \%)$ & 0.007 \\
\hline Pulmonary thromboembolism & $2(4.1 \%)$ & 0 & $2(6.2 \%)$ & \\
\hline Multifocal infarction & $1(2.0 \%)$ & 0 & $1(3.1 \%)$ & \\
\hline Urinary tract infection & $3(6.1 \%)$ & 0 & $3(9.3 \%)$ & \\
\hline Atrial fibrillation & $1(2.0 \%)$ & 0 & $1(3.1 \%)$ & \\
\hline Other & $8(16.3 \%)$ & $1(5.9 \%)$ & $7(21.8 \%)$ & \\
\hline \multicolumn{5}{|l|}{ Clinical outcome } \\
\hline Median hospital stay, days [IQR] & $39.0[9.0 ; 49.0]$ & $8.0[5.0-21.0]$ & $46.5[39.5-52.5]$ & $\begin{array}{l}< \\
0.001\end{array}$ \\
\hline Median intensive care unit stay, days [IQR] & $24.0[2.0 ; 38.0]$ & $0.0[0.0 ; 3.0]$ & $33.5[24.5 ; 41.5]$ & $<0.001$ \\
\hline $\begin{array}{l}\text { Median duration of mechanical ventilation, days } \\
\text { [IQR] }\end{array}$ & - & - & $33.0[22.0-40.5]$ & \\
\hline Tracheostomy & $27(55.1 \%)$ & - & $27(84.4 \%)$ & \\
\hline Ambulation at discharge & $35(71.4 \%)$ & $13(92.9 \%)$ & $22(81.5 \%)$ & 0.609 \\
\hline Death & $5(10.2 \%)$ & $1(5.9 \%)$ & $4(12.5 \%)$ & 0.816 \\
\hline Rehabilitation & 15 (24.5\%) & $1(5.9 \%)$ & $11(34.4 \%)$ & 0.063 \\
\hline Second tetanus vaccination & $24(49.0 \%)$ & $3(17.6 \%)$ & $21(65.6 \%)$ & 0.004 \\
\hline Third tetanus vaccination & $6(12.2 \%)$ & $1(6.2 \%)$ & $5(16.1 \%)$ & 0.617 \\
\hline
\end{tabular}

Abbreviation: $N$ number, $I Q R$ interquartile range

group, and three $(17.7 \%)$ were reported in the nonMV group.

Thirty-two (84.2\%) of the 38 patients admitted to the intensive care unit (ICU) required MV. The median MV duration was 33.0 days (IQR, 22.0-40.5 days), and the hospital stay was longer for the MV group than for the nonMV group (median, 46.5 days [IQR, 39.5-52.5 days] vs. 8.0 days [IQR, $5.0-21.0$ days]; $P<0.001$ ). Eleven patients (34.4\%) in the MV group required rehabilitation, whereas only one patient (5.9\%) in the nonMV group required rehabilitation. The median rehabilitation period was 30.0 days (IQR, 13.0-39.0 days) for the MV group. One patient in the nonMV group underwent rehabilitation for 54.0 days. Most of the patients recovered without impaired motor function after discharge, and the degree of recovery demonstrated no significant difference between the groups $(P=0.609)$. The proportion of secondary tetanus vaccinations was significantly higher in the MV group (65.6\%) than in the nonMV group $(17.6 \% ; P=0.004)$, whereas the proportion of third tetanus vaccinations was not significantly different between the MV (16.1\%) and nonMV groups $(6.2 \% ; P=$ 0.617).

As shown in Table 3, age $>65$ years and the presence of dyspnea were identified as risk factors for the need of MV support in the multivariate regression logistic analysis (age 65 years: odds ratio [OR], 4.63; 95\% confidence interval $[\mathrm{CI}], 1.22-19.99 ; P=0.029$; dyspnea: $\mathrm{OR}, 5.44$; 95\% CI, 1.44-23.43; $P=0.016$ ).

\section{Economic burden and medical costs}

Table 4 shows the economic burden according to the following details: The median total expenditure for the patients with tetanus was US $\$ 18,011$ (range, US \$2680-\$34,497). In particular, the expenditure for the procedure and operation, including MV management, was the most expensive (median, US \$5502), followed by the expenditures for drugs and injections (median, US \$3861) and diagnostic blood tests (median, US \$3764).

Table 3 Multiple logistic regression analysis of risk factors associated with mechanical ventilator support in tetanus patients

\begin{tabular}{|c|c|c|c|c|}
\hline \multirow[t]{2}{*}{ Variable } & \multicolumn{2}{|l|}{ Univariate logistic regression analysis } & \multicolumn{2}{|l|}{ Multivariate logistic regression analysis } \\
\hline & Odds ratio ( $95 \%$ confidence interval) & $P$ value & Odds ratio ( $95 \%$ confidence interval) & $P$ value \\
\hline Female & $0.96(0.28-3.43)$ & 0.949 & - & - \\
\hline Age $>65$ years & $5.28(1.53-20.62)$ & 0.011 & $4.63(1.22-19.99)$ & 0.029 \\
\hline Dyspnea & $6.13(1.76-24.33)$ & 0.006 & $5.44(1.44-23.43)$ & 0.016 \\
\hline
\end{tabular}


Table 4 Medical costs for the 49 inpatients with tetanus

\begin{tabular}{ll}
\hline Factors & Hospitalization costs (USD) \\
\hline Medical costs for treatment, median [IQR] \\
Total cost & $18,011[2680-34,497]$ \\
Cost for patient care & $136[57-247]$ \\
Cost for medications and injections & $3861[603-7114]$ \\
Cost for procedures and operations & $5502[112-8489]$ \\
Cost for diagnostic blood tests & $3764[448-5212]$ \\
Cost for radiological tests & $799[322-1190]$ \\
\hline
\end{tabular}

Abbreviations: IQR interquartile range, USD United States dollars

\section{Clinical outcomes and features by age group}

Table 5 shows the clinical outcomes and features of the older adult patients. Thirty patients were aged $>60$ years, and all of the deceased were in older adult group. The need for MV was significantly greater for those aged $\geq 60$ years than for those aged $<60$ years (24 cases [80.0\%] vs. 8 cases [42.1\%]; $P=0.016$ ). The patients aged $>60$ years had longer hospitalizations than those aged $<$ 60 years (median stay, 40.5 days [IQR, $22.0-51.0$ days] vs. 21.0 days [IQR, 7.0-46.5 days]; $P=0.109$ ).

\section{Discussion}

Although the prevalence of tetanus has decreased with the introduction of tetanus vaccination, tetanus still causes approximately 60,000 deaths per year worldwide [5]. In this study, we reviewed the cases of 49 patients with tetanus in two tertiary referral centers. The median age of the 49 patients with tetanus was 67.0 years; of the patients, five died and $65.3 \%$ required MV. The median hospital stay of the patients with tetanus was 39.0 days. The older adult patients required more MV, longer-term hospital care, and higher treatment cost than the young patients.

This study is comparable with previous studies in terms of the patients' clinical characteristics. Most of the patients with tetanus were older adults, a similar finding to previous reports in developed countries [1, 6-8]. The female predominance among the patients with tetanus was consistent with the results from France and Italy, possibly due to the essential immunizations administered to men during their compulsory military service
$[6,9,10]$. In our study, the most common entry site was unknown, which was comparable with other reports [11, 12]. However, other studies have reported that $90 \%$ of patients were aware of the entry site $[8,13,14]$. It is possible that the wound was too small for the patients to discover or was chronic and therefore overlooked given that an acute traumatic wound was the primary focus.

Five of our patients died during the study, resulting in a $10.2 \%$ mortality rate, which was comparable with the 13.2 and $16.5 \%$ in studies in the United States and Italy, respectively $[1,6]$. A previous study in France showed a relatively high mortality rate of up to $44.5 \%$ due to the inclusion of patients with severe tetanus who were admitted to the ICU [15]. In the present study, the clinical outcomes were consistent with those of a previously published national report in Japan [7]. Nakajima et al. showed that $53.5 \%$ of all patients required MV and that most deaths occurred in the older adult group, which could be explained by the similarity in the healthcare insurance system and medical accessibility between the two countries [16]. Studies have shown that tetanus severity is associated with older adult patients, even for different healthcare insurance systems. In the United States, age $>65$ years was associated with a higher risk of fatal tetanus (OR, 9.6; 95\% CI, 3.6-25) [1]. In a French study of older adult ICU patients with tetanus (median age, 80 years), only age was related to the 1 -year mortality rate (mean age of the deceased vs. mean age of the survivors at 1 year: 83 years [range, $81-85$ years] vs. 79 years [range, $73-84$ years]; $P=0.03$ ) [17]. As in previous reports, our results showed higher MV and mortality rates in the older adult group. This study of tetanus patients demonstrates that older age and dyspnea may be the cause of the tendency toward MV support in the multivariate logistic regression analysis, but we did not find any studies explaining the risk factor related to MV support in patients with tetanus. We thought that further research would need to define the risk factors of mortality through a multivariate adjusted method.

Our study showed relatively lower healthcare costs for tetanus than those reported in previous studies [18-20]. However, considering that the healthcare expenditures have been relatively lower in South Korea than in other

Table $\mathbf{5}$ Clinical outcomes of the patients with tetanus by age

\begin{tabular}{|c|c|c|c|}
\hline Outcome & Age $<60$ years $(n=19)$ & Age $\geq 60$ years $(n=30)$ & $P$ value \\
\hline Death, n (\%) & 0 & $5(16.7 \%)$ & \\
\hline Need for mechanical ventilation, $n(\%)$ & $8(42.1 \%)$ & $24(80.0 \%)$ & 0.016 \\
\hline Intensive care unit stay, median [IQR] (days) & $3.0[0.0 ; 35.0]$ & $30.0[9.0 ; 39.0]$ & 0.117 \\
\hline Duration of mechanical ventilation, median [IQR] (days) & $0.0[0.0 ; 31.0]$ & $28.5[7.0 ; 38.0]$ & 0.080 \\
\hline Hospital stay, days [IQR] (days) & $21.0[7.0 ; 46.5]$ & $40.5[22.0 ; 51.0]$ & 0.109 \\
\hline Total medical costs, median [IQR] (USD) & $1634.8[530.2 ; 9712.6]$ & $4906.5[1690.1 ; 9100.1]$ & 0.303 \\
\hline
\end{tabular}

Abbreviations: $N$ number, IQR interquartile range, USD United States dollars 
countries, our results were consistent with previous reports in that the individual economic burden from tetanus was great. Previous data suggested that the annual expenditures in 2018 for inpatient curative and rehabilitative care were US \$582.4 per person in South Korea, US $\$ 1210.1$ in Japan, and US $\$ 1752.4$ in the United States [21]. In our study, the amount of US $\$ 18,011$ per inpatient with tetanus exceeded by threefold the annual health expenditure per person in South Korea. When compared with the medical expenditures per person for other diseases in South Korea (e.g., US \$3966.3 for sepsis, US $\$ 5230.8$ for cardiac disease, and US $\$ 7352.5$ for stomach cancer, as indicated by the Health Insurance Review and Assessment Service), the direct medical expenditures for inpatients with tetanus were significantly more expensive [22]. This does not even take into account the indirect costs of the disease, such as lost wages and productivity.

To our knowledge, no studies have reported on the Tdap or Td vaccination rate in adults in South Korea. A previous report indicated that the estimated DTaP coverage among children aged $<7$ years with five vaccinations was $56.6 \%$, despite that the Korean National Immunization Program has financed the DTaP immunization, [23]. Other reports have shown that the proportion of antitetanus immunoglobulin-G titers $\geq 0.1$ $\mathrm{IU} / \mathrm{mL}$ steadily decreased in the older adult group and that the tetanus seroprevalence rate was $19.3 \%$ for the age group $>60$ years in Korea $[9,24]$. The US National Center for Health Statistics in 2015 released a report that the tetanus vaccination rate for adults aged $>65$ years was $56.9 \%$ over the past 10 years in the United States [25]. Choi et al. reported that all groups aged > 40 years responded with $>0.1-\mathrm{U} / \mathrm{mL}$ tetanus antibody levels after the third vaccine dose [26]. Our study showed a low vaccination rate even in patients who had experienced tetanus. Considering that the cost of three doses of the Td vaccine in South Korea is US \$72 and the extraordinarily high medical cost of treating tetanus of US $\$ 18,010.5$, a massive financial incentive is allotted to ensuring sufficient $\mathrm{Td}$ vaccination, thereby preventing tetanus and its consequent medical costs. We believe that further studies regarding the status of Tdap or $\mathrm{Td}$ vaccination in adults will be helpful to highlight the advantages of vaccination.

This study has a number of limitations. First, it was conducted at two local tertiary referral hospitals. Given that approximately 20 tetanus cases are reported annually in South Korea by the KCDC, the data in this study should be interpreted with caution because the cases represented approximately $20 \%$ of all tetanus cases in South Korea. Nevertheless, we believe that our data are noteworthy because the two tertiary university hospitals are national hospitals in Gyeongsangbuk-do, Jeollabuk- do, and a representative province in South Korea. Collecting data from a large number of cases would be helpful to further define the clinical manifestations and risk factors of mortality through a multivariate adjusted method. Second, the quality of the data depends on the historical data available in this retrospective cohort study. Third, we did not include healthcare costs and additional indirect costs, such as private nursing care and lost earnings during hospitalization. Nevertheless, our study is one of the few studies that have investigated the costs of treatment and economic burden caused by tetanus, and provides noteworthy information given the rarity of tetanus, which, to our knowledge, has not been investigated in a tertiary university hospital in the past 10 years.

\section{Conclusion}

In summary, tetanus remains a severe but preventable acute infection disease, and its treatment entails high medical costs, which could be challenging for many individuals. The study observed a high rate of MV and high medical costs due to the intensive care required for older adult patients; however, tetanus immunization in adults is often overlooked. Therefore, strategies to improve tetanus vaccination should be implemented, especially for older adults.

\section{Abbreviations}

USD: United States dollars; Tdap: Toxoids and diphtheria and acellular pertussis vaccine; Td: Tetanus toxoids vaccine; SD: Standard deviation; IQR: Interquartile range; ICU: Intensive care unit; MV: Mechanical ventilation; SBP: Systolic blood pressure; OR: Odds ratio; Cl: Confidence interval

\section{Acknowledgments}

Abstract of these data was presented in IDweek 2020 (absrt.1552), and published in open forum infectious diseases, volume 7, issue supplement_1, October 2020, page S777

\section{Authors' contributions}

Conceptualization: HHC and SIJ. Methodology: SWK. Formal analysis and data curation: HHC, SWK, and SIJ. Validation: YK. Investigation: MG, KTK, and SH. Writing - original draft preparation: SB and HHC. Writing - review and editing: HHC and SIJ. Approval of final manuscript: All authors. The authors read and approved the final manuscript.

\section{Funding}

This study was conducted with no external funding.

\section{Availability of data and materials \\ The datasets used during the present study are available from the corresponding author on reasonable request.}

\section{Declarations}

\section{Ethics approval and consent to participate}

The study was approved by the institutional review board of Kyungpook National University Hospital and Chonnam National University Hospital (approval Nos. KNUH-201907031 and CNUH-2019235, respectively). Written informed consent was waived owing to the retrospective nature of the study. The data used in this study were anonymized before use.

Consent for publication

Not applicable. 


\section{Competing interests}

There are no competing interests to declare.

\section{Author details}

'Division of Infectious Diseases, Department of Internal Medicine, School of Medicine, Kyungpook National University, 130 Dongdeok-ro, Jung-gu, Daegu 41944, South Korea. ${ }^{2}$ Department of Internal Medicine, Kyungpook National University Hospital, Daegu, South Korea. ${ }^{3}$ Department of Infectious Diseases, Chonnam National University Medical School, 42 Jebongro, Donggu, Gwangju 61469, South Korea.

\section{Received: 26 October 2020 Accepted: 25 February 2021}

Published online: 09 March 2021

\section{References}

1. Control CD. Prevention: tetanus surveillance---United States, 2001-2008. MMWR Morb Mortal Wkly Rep. 2011;60(12):365.

2. Yoon Y-H, Moon S-W, Choi S-H, Cho Y-D, Kim J-Y, Kwak YH. Clinician awareness of tetanus-diphtheria vaccination in trauma patients: a questionnaire study. Scand J Trauma Resusc Emerg Med. 2012;20(1):35.

3. Choi EH, Park SE, Kim Y-J, Jo DS, Kim Y-K, Eun B-W, Lee T-J, Lee J, Lee H, Kim $\mathrm{KH}$. Recommended immunization schedule for children and adolescents: committee on infectious diseases of the Korean pediatric society, 2018. Korean J Pediatr. 2019;62(7):252-6.

4. Kerr JH, Corbett $J$, Spalding JM. Sympathetic overactivity in severe tetanus. Proc R Soc Med. 1969;62(7):659-62

5. Wang H, Naghavi M, Allen C, Barber RM, Bhutta ZA, Carter A, Casey DC, Charlson FJ, Chen AZ, Coates MM. Global, regional, and national life expectancy, all-cause mortality, and cause-specific mortality for 249 causes of death, 1980-2015: a systematic analysis for the global burden of disease study 2015. Lancet. 2016;388(10053):1459-544.

6. Filia A, Bella A, von Hunolstein C, Pinto A, Alfarone G, Declich S, Rota MC. Tetanus in Italy 2001-2010: a continuing threat in older adults. Vaccine. 2014;32(6):639-44.

7. Nakajima M, Aso S, Matsui $H$, Fushimi $K$, Yasunaga $H$. Clinical features and outcomes of tetanus: analysis using a National Inpatient Database in Japan. J Crit Care. 2018:44:388-91.

8. Shin DH, Yu HS, Park JH, Shin JH, Kim SJ. Recently occurring adult tetanus in Korea: emphasis on immunization and awareness of tetanus. J Korean Med Sci. 2003;18(1):11.

9. Kang JH, Hur JK, Kim JH, Lee Kl, Park SE, Huk S, Lee MS, Ban SJ, Hong SH, $\mathrm{Cho} \mathrm{DH}$. Age related serosurvey of immunity to tetanus in Korean populations. Korean J Infect Dis. 2001;33(2):104-11.

10. Gidding HF, Backhouse JL, Gilbert GL, Burgess MA. Immunity to diphtheria and tetanus in Australia: a national serosurvey. Med J Aust. 2005;183(6):3014.

11. Yen LM, Thwaites CL. Tetanus. Lancet. 2019;393(10181):1657-68.

12. Thwaites $C L$, Yen LM, Nga NT, Parry J, Binh NT, Loan HT, Thuy TT, Bethell D, Parry CM, White NJ, et al. Impact of improved vaccination programme and intensive care facilities on incidence and outcome of tetanus in southern Vietnam, 1993-2002. Trans R Soc Trop Med Hyg. 2004;98(11):671-7.

13. Malani PN. Mandell, Douglas, and Bennett's principles and practice of infectious diseases. JAMA. 2010;304(18):2067-71.

14. Ergonul O, Egeli D, Kahyaoglu B, Bahar M, Etienne M, Bleck T. An unexpected tetanus case. Lancet Infect Dis. 2016;16(6):746-52.

15. da Nóbrega MVD, Reis RC, Aguiar ICV, Queiroz TV, Lima ACF, Pereira EDB, de Araujo Ferreira RF. Patients with severe accidental tetanus admitted to an intensive care unit in northeastern Brazil: clinical-epidemiological profile and risk factors for mortality. Braz J Infect Dis. 2016;20(5):457-61.

16. Dronina Y, Yoon YM, Sakamaki H, Nam EW. Health system development and performance in Korea and Japan: a comparative study of 2000-2013. J Lifestyle Med. 2016;6(1):16-26.

17. Mahieu R, Reydel T, Maamar A, Tadié J-M, Jamet A, Thille AW, Chudeau N, Huntzinger J, Grangé S, Beduneau G. Admission of tetanus patients to the ICU: a retrospective multicentre study. Ann Intensive Care. 2017;7(1):1-7.

18. Thuy DB, Campbell Jl, Thanh TT, Thuy CT, Loan HT, Hao NV, Minh YL, Tan LV, Boni MF, Thwaites CL. Tetanus in southern Vietnam: current situation. Am J Trop Med Hyg. 2017;96(1):93-6.

19. Guzman-Cottrill JA, Lancioni C, Eriksson C, Cho YJ, Liko J. Notes from the field: tetanus in an unvaccinated child - Oregon, 2017. MMWR Morb Mortal Wkly Rep. 2019;68(9):231-2.
20. Yen C, Murray E, Zipprich J, Winter K, Harriman K. Missed opportunities for tetanus postexposure prophylaxis--California, January 2008-march 2014 MMWR Morb Mortal Wkly Rep. 2015;64(9):243-6.

21. Joint OECD, EUROSTAT and WHO Health Accounts SHA Questionnaires (JHAQ). https://stats.oecd.org/Index.aspx?DatasetCode=HEALTH_STAT 2021 Accessed 11 Jan 2021.

22. Health Bigdata. Hub http://opendata.hira.or.kr/op/opc/olapMfrnIntrs/lnslnfo. do 2021. Accessed 11 Jan 2021.

23. Choe YJ, Yang JJ, Park SK, Choi EH, Lee HJ. Comparative estimation of coverage between national immunization program vaccines and non-NIP vaccines in Korea. J Korean Med Sci. 2013;28(9):1283-8.

24. Sung H, Jang MJ, Bae EY, Han SB, Kim J-H, Kang JH, Park Y-J, Ma SH. Seroepidemiology of tetanus in Korean adults and adolescents in 2012. J Infect Chemother. 2014;20(7):397-400.

25. Norris T, Vahratian A, Cohen RA. Vaccination coverage among adults aged 65 and over: United States. NCHS Data Brief. 2015;2017(281):1-8.

26. Choi J-H, Choo EJ, Huh A, Choi S-M, Eom JS, Lee JS, Park SH, Kang JH. Immunogenicity and safety of diphtheria-tetanus vaccine in adults. J Korean Med Sci. 2010;25(12):1727-32.

\section{Publisher's Note}

Springer Nature remains neutral with regard to jurisdictional claims in published maps and institutional affiliations.
Ready to submit your research? Choose BMC and benefit from:

- fast, convenient online submission

- thorough peer review by experienced researchers in your field

- rapid publication on acceptance

- support for research data, including large and complex data types

- gold Open Access which fosters wider collaboration and increased citations

- maximum visibility for your research: over $100 \mathrm{M}$ website views per year

At BMC, research is always in progress.

Learn more biomedcentral.com/submissions 